\title{
Parietal theta burst TMS: functional fractionation observed during bistable perception not evident in attention tasks
}

\author{
Georg Schauer $^{1,2}$, Ryota Kanai ${ }^{1,3}$ and Jan Willem Brascamp ${ }^{4}$ \\ ${ }^{1}$ Sackler Centre for Consciousness Science, University of Sussex, Brighton, Sussex, UK. \\ ${ }^{2}$ Centre for Integrative Neuroscience, University of Tübingen, Tübingen, Germany \\ ${ }^{3}$ ARAYA Brain Imaging, Tokyo, Japan \\ ${ }^{4}$ Department of Psychology, Michigan State University, East Lansing, Michigan, USA.
}

Corresponding Author:

Georg Schauer

info@georgschauer.com

Address at which the study was carried out:

Sackler Centre for Consciousness Science

Chichester I, University of Sussex

Brighton, BN1 9RH

United Kingdom

Present work address of corresponding author:

Vision and Cognition Lab, Centre for Integrative Neuroscience

University of Tübingen

Otfried-Müller-Straße 25

72076 Tübingen

Germany

\begin{abstract}
(149 words)
When visual input is ambiguous, perception spontaneously alternates between interpretations: bistable perception. Studies have identified two distinct sites near the right intraparietal sulcus where inhibitory transcranial magnetic stimulation (TMS) affects the frequency of occurrence of these alternations, but strikingly with opposite directions of effect for the two sites. Lesion and TMS studies on spatial and sustained attention have also indicated a parcellation of right parietal cortex, into areas serving distinct attentional functions. We used the exact TMS procedure previously employed to affect bistable perception, yet measured its effect on spatial and sustained attention tasks. Although there was a trend for TMS to affect performance, trends were consistently similar for both parietal sites, with no indication of opposite effects. We interpret this as signifying that the previously observed parietal fractionation of function regarding the perception of ambiguous stimuli is not due to TMS-induced modification of spatial or sustained attention.
\end{abstract}

Keywords: Spatial attention, sustained attention, parietal cortex, TMS, bistable perception 


\section{Introduction}

The functional role of the right parietal cortex in consciousness has long been subject to debate (Lumer et al., 1998; Kleinschmidt et al., 1998; Rees et al., 2002, Knapen et al., 2011; Weilnhammer et al., 2013; Watanabe et al., 2014; Brascamp et al., 2015). A recent contribution to this debate has come from several studies that combined transcranial magnetic stimulation (TMS) with bistable perception paradigms (Kanai et al., 2010, 2011; Carmel et al., 2010; Zaretskaya et al., 2010, 2013; Wood et al., in prep). In these paradigms, participants view a stimulus that is ambiguous or internally conflicting, leading to periodic fluctuations in the conscious perception of that stimulus over time, even though the stimulus is unchanging. In the present work we focus on one particular set of findings from recent studies that used inhibitory TMS. First, Carmel et al. (2010) and Kanai et al. (2011) used inhibitory TMS to the right anterior superior parietal lobule (ant-SPLr) at coordinates previously indicated in a neuroimaging study on bistable perception (Lumer et al., 1998) and found that perception fluctuated more frequently as a result. In other words, inhibitory TMS to this location appeared to destabilise the percept. Interestingly, inhibitory TMS on a more posterior portion of the right superior parietal lobule (post-SPLr), identified based on acrossparticipant correlations between anatomy and perception of ambiguous stimuli, had the opposite effect of reducing the rate of switching between perceptual interpretations (Kanai et al., 2010).

One proposed interpretation of this functional fractionation of the right parietal cortex is that the ant-SPLr is associated with top-down predictions of oncoming sensory stimulation, while post-SPLr processes the error signals that arise from comparing these predictions to actual input (Kanai et al., 2011). In the current paper we test an alternative interpretation. Specifically, the perceptual switch rate during bistable perception is known to be influenced by the allocation of attention to the ambiguous stimulus (Paffen et al., 2006; Alais et al., 2010; Pastukhov \& Braun, 2007), and numerous reports show parietal impairment to be associated with altered attention function (e.g. Malhotra et al., 2009; Hilgetag et al., 2001; Thut et al.,2005; Rueckert \& Grafman, 1998; Hodsoll et al., 2009; Rushworth et al., 2006). This raises the question of whether TMS-induced alterations in attention allocation could explain the observed effects of parietal TMS on bistable perception. Under this account, the altered switch rate may result from inhibition of brain areas essential for maintaining attention, potentially leading to shorter percept durations, and for producing attentional shifts, potentially leading to longer percept durations. The functional fractionation of the right parietal cortex during bistable perception could hence represent a parcellation into separate attention modules rather than regions that can specifically be associated with prediction and error signal processing.

Aside from the known effect of attention allocation on bistable perception, other evidence also lends credence to this alternative account. Independent of the above mentioned work on bistable perception, a functional parcellation of right parietal cortex is suggested by work that shows superior regions to be associated with attentional shifts and spatial attention (Yantis et al., 2002; Vandenberghe \& Gillebert, 2009; Müller-Plath et al., 2010), and work that implicates more inferior regions in sustained attention and non-spatial tasks (Pardo et al., 1991; Husain \& Rorden, 2003, Kelley et al., 2007; Malhotra et al., 2009; review in Vandenberghe et al., 2012); a separation that is approximately consistent with the distinction between the more superior ant-SPLr and the more inferior post-SPLr. Support for this model has primarily come from lesion studies on neglect and sustained attention summarised below.

A common attention deficit associated with right parietal lesions is left hemispatial neglect, which is a disorder characterised by a lateralised visual information processing bias, which is not due to abnormal early sensory perception, but rather comes from an inability to attribute saliency to stimuli 
within the contralesional visual field, or to take notice of information in the affected sensory space (Mesulam, 1981, 1985). While damage to either hemisphere can lead to neglect of contralesional space (Heilman et al., 1983), the effect is materially stronger for right-parietal lesions (Heilman \& van den Abell, 1980; Critchley, 1953), causing more severe and long-lasting deficits (Stone et al., 1993). For instance, patients have trouble in eating from the left side of their plates or reading a clock when its hands are on its left half as if their attention is permanently shifted away from the left and almost "magnetically attracted" to the right side of their visual field (Gainotti et al., 1991).

Several studies have transiently elicited spatial attention effects that resemble neglect, by applying TMS to the right parietal cortex of healthy participants (e.g. Fierro et al., 2000; Bjoertomt et al., 2002; Hilgetag et al., 2001). With regard to our current question considering a potential role of attention in mediating the effect of TMS on bistable perception, it is relevant to ask whether the induction of these spatial attention effects is dependent on the exact location of parietal stimulation. In particular, what is the effect of inhibitory TMS on lateralised spatial attention specifically at the parietal loci indicated by stimulation studies on bistable perception (Carmel, et al., 2010; Kanai, et al., 2010)? The existing literature is agnostic towards whether the precise localisation of the TMS site will yield a different result, in part because the relevant studies on lateralised spatial attention, being somewhat older, selected their stimulation sites based on EEG electrode positions (Fierro et al., 2000; Hilgetag et al., 2001) and functional tests (Bjoertomt et al., 2002), whereas the more recent studies on bistable perception characterised their stimulation sites in terms of standard brain coordinates, using neuronavigation based on participants' individual MRI scans (Kanai, et al., 2010, 2011; Carmel, et al., 2010). Reliably translating between these various localisation methods is not straightforward (Rushworth, et al., 2001; Rushworth, et al., 2006; Sack, et al., 2009), making it difficult to assert whether the test sites in the previous literature on lateralised spatial attention overlap with the precise test sites in the literature on bistable perception.

A second attentional function in which we were interested because of proven links to right parietal cortex and, moreover, a plausible relation to the bistable perception findings, was sustained attention, or vigilance (e.g. Adler et al., Synapse 2001; Rueckert \& Grafman, 1998; Sarter et al, 2001; Whyte et al., 1995, Malhotra et al., 2009). This refers to the ability to maintain good performance on tasks requiring attention over prolonged periods of time. A relation to the bistable perception findings is conceivable in the sense that the perceptual switch rate is known to depend on the amount of attention paid to the stimulus inducing bistable perception (Paffen et al., 2006; Alais et al., 2010; Pastukhov \& Braun, 2007), so that any TMS-induced change in this amount could lead to altered switch rates. Several studies link sustained attention to right parietal cortex, but evidence does not consistently point to any specific subregion (Malhotra et al., 2009; Serences \& Yantis, 2007; Thakral \& Slotnick, 2009).

In the current study, we asked whether the fractionation of parietal cortex in the conscious perception of bistable stimuli reflects a fractionation in function pertaining to spatial and sustained attention. Existing studies do not allow this assertion, in part due to the difficulty of drawing up a precise functional anatomy of attention based on results from lesion studies and from TMS studies without accurate neuronavigation. The solution we chose in this study was to replicate the exact localisation procedures that we previously used in TMS studies that showed two opposite effects on bistable perception (Kanai et al., 2010; Kanai et al., 2011; Wood et al., in prep), but to measure effects on tasks pertaining to spatial attention (identifying dots in the visual periphery, Hilgetag et al., 2001) and sustained attention (withholding response based on spatial location, Malhotra et al., 2009). We theorised that the functional dissociation between anterior and posterior parietal cortex that is evident in bistable perception, may also show in these attention tasks. Specifically, we 
hypothesised that inhibitory TMS to ant-SPLr would replicate the finding of decreased performance on a task requiring attention to space contralateral to TMS stimulation coupled with improved performance in ipsilateral space, as found in Hilgetag (et al., 2001). We also hypothesised that inhibitory TMS to post-SPLr would lead to sustained attention deficits over time, as observed in Malhotra (et al., 2009). This would entail that the fractionation into ant-SPLr and post-SPLr observed in bistable perception at least in part overlaps with the fractionation between superior parietal cortex being associated with attentional shifts/spatial attention and inferior parietal cortex being associated with sustained attention (Vandenberghe et al., 2012). Such an overlap would open the door to several interpretations of how parietal cortex function relates to bistability and attention. For instance, if post-SPLr is associated with sustained attention, as we hypothesise, then inhibition of post-SPLr should have led to a decrease in bistable dominance durations in Kanai et al. (2010), which it did not. However, withdrawing attention from a bistable perception stimulus has been shown to increase percept durations (Paffen et al., 2006), which would suggest that inhibiting sustained attention related areas ought to lead to an increase in rivalry dominance. It is also conceivable that removing attentional resources lowers the likelihood of the perceptual system reselecting alternative interpretations of the ambiguous input. In summary, it is unclear what the role of attention is in the conscious perception of ambiguous stimuli and moreover how this relationship is mediated by the right parietal cortex. 


\section{Material and methods}

\subsection{Participants}

12 healthy volunteers with normal or corrected to normal vision were recruited for a fully counterbalanced design (6 TMS target orders $\times 2$ task orders). One participant was not able to reliably see one of the stimuli, hence a new participant was recruited in her stead (final sample: age 21-37 yrs, mean 26.42 yrs \pm 5.70 S.D.; 4 female, 9 right handed). Another participant's MRI scan displayed an anatomically abnormal $3 \mathrm{~mm}$ wide cavity in his right-frontal white matter, which was deemed clinically irrelevant by the attending neuroradiologist and the participant was subsequently recruited. Written informed consent was acquired from all participants prior to testing, which was approved by the university's local ethics committee. Following consent, participants were screened to ensure that they met health criteria for inclusion in a theta burst TMS study.

\subsection{Visual stimuli and apparatus}
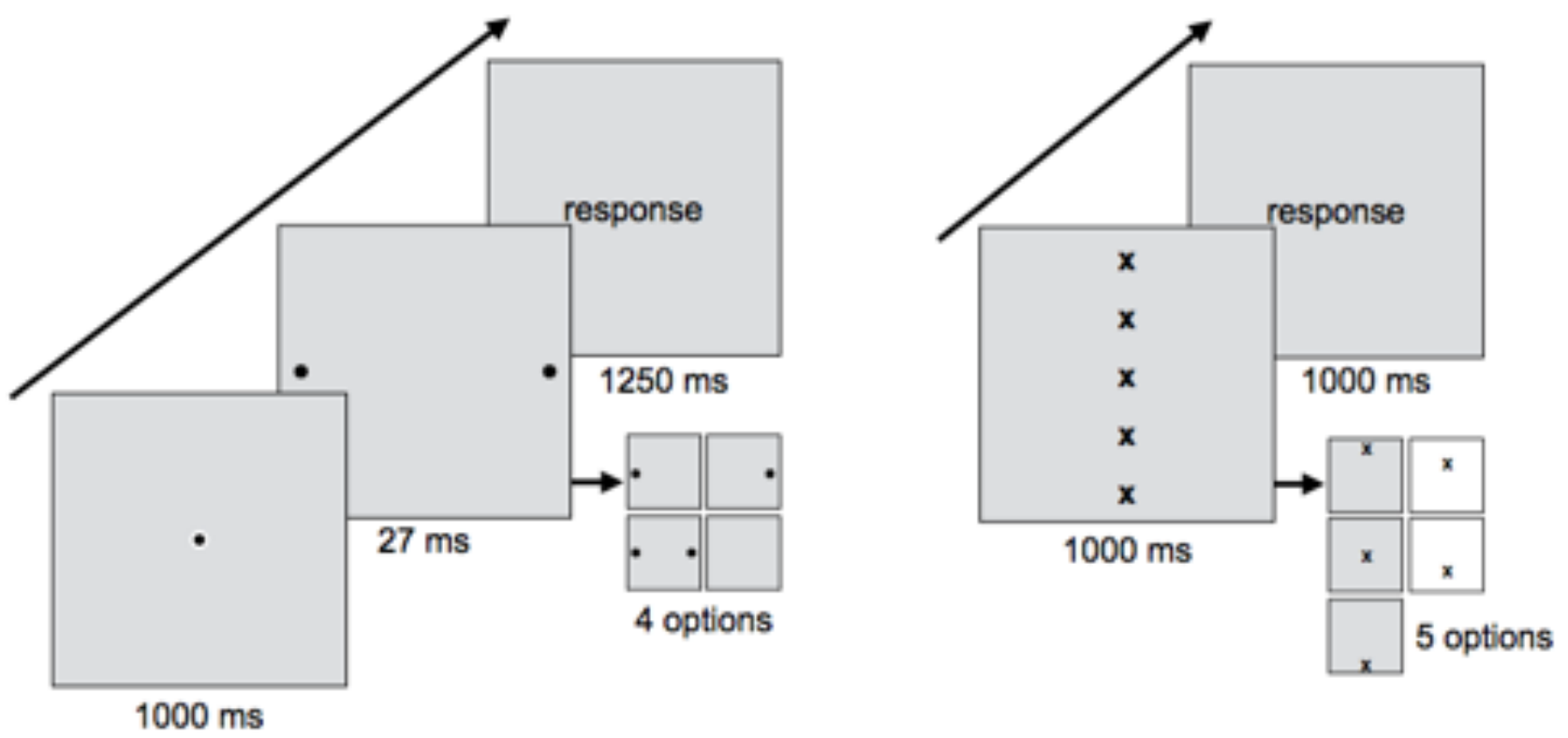

Figure 1: Psychophysical paradigm for task 1 (I) and task 2 (II)

Task 1 - Lateralised Spatial Attention. Participants were seated before a grey screen and instructed to fixate on a central dot. After starting the experiment with a button press, participants were presented with small black dots of various sizes (see section on experimental design) in the periphery of their visual field at 24 degrees left and right to the central dot (Figure 1, left). Each trial started with the central dot in isolation, and following $1000 \mathrm{~ms}$ of fixation either a single dot appeared in the far right visual field of the participant, or it appeared on the left, or two dots appeared bilaterally or no dot appeared at all. Dots were present for $40 \mathrm{~ms}$. Following dot presentation, participants had $1250 \mathrm{~ms}$ to make a 4-AFC between the four options using the number pad on a keyboard. Overall, there were 60 trials of left-lateralised dots, 60 trials of right-lateralised dots, 60 bilateral presentations and 30 trials in which no dot was presented at all. Total task duration 
was just over 8 minutes. This task largely replicates the procedure used in Hilgetag et al. (2002), and is meant to index deficits in lateralised spatial attention.

Task 2 - Sustained Attention. Participants were seated before a grey screen and instructed to fixate on the centre of the display. After starting the experiment with a button press, participants were presented with a black cross, 1.7 angular degrees in diameter, in one of five predefined locations, evenly spaced along the vertical meridian of the participant's field of view on the screen, ranging from 12 degrees above to 12 degrees below the centre of the screen. The five locations were explicitly shown to the participant prior to the experiment, but not during the task. Participants were instructed to press a button as quickly as possible if a cross appeared in either the second or fourth location from the top, or do nothing in case a cross appeared at any other location. The proportion of trials requiring a response was determined for each participant individually (see section on experimental design). For each trial, a cross in one of the five positions was presented for $1000 \mathrm{~ms}$, followed by $1000 \mathrm{~ms}$ of pause. Overall, there were 300 trials. The total task duration was about 10 minutes. This task largely replicates experiment 2 in Malhotra et al. (2009), and is meant to index deficits in sustained attention. Since targets appear in distinct locations along the vertical meridian, there is a spatial component to this task. However, while task 1 indexes attentional performance in the detection of visual stimuli in the visual periphery, the targets in task 2 are vertically centred, making the spatial components of both tasks independent.

All stimuli were displayed on a 19 inch Lacie CRT monitor (75hz refreshing rate at $1024 \times 768$ pixel resolution; Lacie, Paris, France) controlled by a Dell Precision PC on Windows XP, using the Psychtoolbox package for MATLAB R2014b. There was no natural light contamination nor room lighting. A chin rest was used to minimise head movements. All participants used their right hand for button presses.

\subsection{Experimental design}

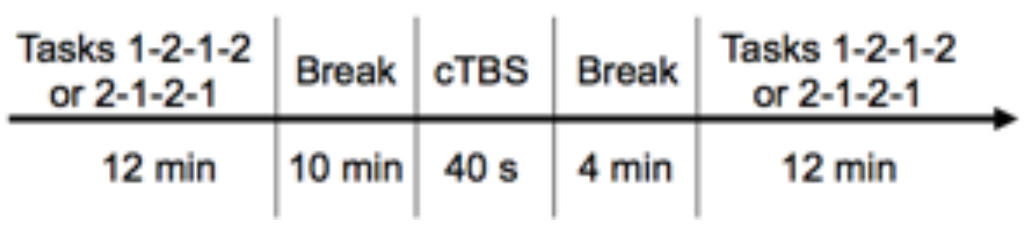

Figure 2: Experimental procedure for days 2, 3 and 4

Participants came to the lab on four separate occasions, at least 24 hours apart to prevent TMS carry over effects. On the first day, participants were made familiar with the two behavioural tasks administered during the other three occasions that involved TMS, and at the same time the data from this day was used to individualise the stimuli for each participant. Specifically, participants completed three runs of 120 trials of task 1 with five different dot sizes. Following each run, we visually examined the data for floor or ceiling effects and adjusted the task difficulty via the upper and lower bound of dot sizes for the next run: the smaller the dots, the more difficult the task. After completion of this pre-experiment, we chose two dot sizes to be used during the TMS sessions for each participant, which ensured that participants' performance would not be saturated with all 
correct / incorrect responses. We aimed for participants correctly responding to more than $30 \%$ of targets for the small dot and less than $70 \%$ for the large dot (mean small dot size: 0.09 angular degrees \pm 0.039 S.D.; mean large dot size: 0.13 angular degrees \pm 0.054 S.D.). Following previous work (Hilgetag et al., 2001) both target sizes were randomly interleaved during the actual experiment, and performance was averaged across both sizes. The pre-experiment lasted about 30 minutes. To avoid floor effects in task 2, we manipulated the frequency at which response trials appeared. Participants completed four runs of 40 trials each. During each run, crosses appeared at the response locations on either $40 \%, 50 \%, 60 \%$ or $70 \%$ of trials in random order. Given participants' propensity to make more, unwarranted, responses on no-response trials (false alarms) when the frequency of response trials is higher, we chose a frequency that ensured that participants made an adequate number of such false alarms (more than 10\% of no-response trials). This frequency was then used for task 2 in the TMS sessions. This pre-experiment lasted about 20 minutes and the average percentage of response trials was $68 \%$ ( \pm 0.04 S.D.) across participants.

The sequence of events on each of days 2, 3 and 4 is shown in figure 2. Participants first completed two runs of each of the behavioural tasks. For task 1, 86 trials were performed ( 8 absent targets and 13 trials for each target location times 2 dot sizes), which took about 3.3 minutes. For task 2, 80 trials were performed, which required about 2.6 minutes. Both tasks were interleaved, so that each participant ran the two tasks either in order 1, 2, 1, 2, or in order 2, 1,2, 1 . This took about 12 minutes in total. After task completion, there was a 10 minute break, during which the neuronavigation system was set up. Afterwards, there were 40 seconds of inhibitory theta burst TMS to one of three anatomical sites. After stimulation, there was a 4 minute break, followed by a repetition of the behavioural tasks that the participant had performed before TMS, again in the same order. This procedure was performed three times on separate days, once for each of the three TMS target sites. The order in which the sites were targeted, as well as the order in which the two behavioural tasks were interleaved, was counterbalanced across participants (see section below).

\subsection{TMS protocol and neuronavigation}

The TMS protocol was identical to the one used in Kanai et al. $(2010,2011)$. A burst of three $50 \mathrm{~Hz}$ TMS pulses were applied every $200 \mathrm{~ms}$ for 40 seconds, at an intensity of $40 \%$ of the maximum stimulator output. On separate days, these pulses were applied either to the post-SPLr (MNI: $x=38$, $y=-64, z=32)$, to the ant-SPLr (MNI: $x=36, y=-45, z=51)$, or to the control site vertex. TMS pulses were delivered using a Magstim Rapid 2 figure of 8 alpha coated coil (70mm coil width, maximum field strength $=2.2 \mathrm{~T}$, Magstim Ltd, Whitland, United Kingdom). Participants did not drink alcohol in the $24 \mathrm{hrs}$ prior to each session and were well rested (both to avoid risk of lowered seizure threshold, see Rossi et al., 2009). 


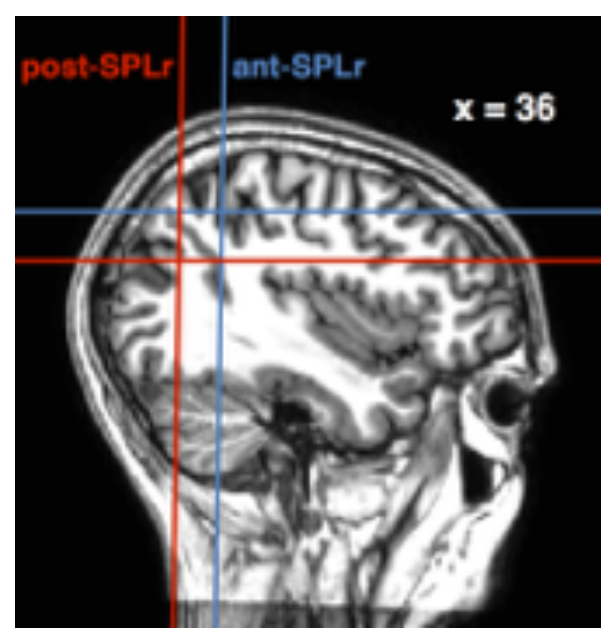

Figure 3: ant-SPLr and post-SPLr on the T1 MRI scan of a representative participant, MRI $\mathrm{x}$ axis position is an approximation of the two regions for visualisation purposes.

The two parietal locations were localised with standard MNI brain coordinates on the basis of each participant's anatomical MRI scan using the neuronavigation system Visor2 (ANT, Enschede, the Netherlands) with an tracking system using a Polaris infra-red camera (Northern Digital, Waterloo, Canada). The coil was held manually by the experimenter with its shaft pointed directly downwards at a $90^{\circ}$ angle from the floor. The distance between actual coil location and its optimal positioning was kept at less than $1.5 \mathrm{~mm}$ at all times during stimulation. The vertex of the participant was localized using externally visible anatomical landmarks. Using flexible measuring tape, the midpoint of the medial line on the scalp between nasion and inion was marked on the swim cap, which is directly superior to the vertex. For vertex stimulation the coil was held against the participant's scalp with its handle pointing straight behind the participant, with the experimenter standing behind the participant and holding the coil parallel to the floor.

\subsection{Data analysis}

Our main measure of interest is the change in task performance following TMS, found by comparing the behavioural results post-TMS to pre-TMS for each testing session. The reason that we recorded a new baseline of performance each session is to control for day to day variations in, for instance, arousal and attention, that may give rise to between-day performance differences. Any pre-post difference in behavioural performance for TMS to the vertex was assumed to reflect TMS site-unspecific effects on the brain and was subtracted from the difference in performance found for the other TMS sites to find the site-specific effect of parietal TMS on behavioural performance. For task 1, we examined the proportion of correctly identified targets on the right, on the left, bilaterally, as well as the tendency to only report a left or right dot when, in fact, dots were presented bilaterally. For task 2, we examined the false alarm rate, i.e. the proportion of responses to noresponse trials, as well as changes in this rate over time. 


\section{Results}

\subsection{Task 1 - Spatial attention}

Given the expectation that TMS may affect performance on this task, we first turned to each session day's pre-TMS data to verify whether participants were able to complete the task. For trials on which no target was presented, participants successfully withheld their response in $86 \%$ of cases, suggesting they were performing the task as intended. The results for trials on which there actually was a target, provide further reassurance. Previous work has shown that observers seldom report the location of a unilateral target as opposite to its actual location, and that bilateral targets are more often misidentified as unilaterally left than as unilaterally right (Hilgetag et al., 2001). Our results, summarised in Figure 4, are consistent with this. Unilateral targets were essentially never reported as being on the location opposite their actual location, and there was a tendency to more often identify bilateral targets as unilaterally left ( $18 \%$ of trials on average across observers) than as unilaterally right (15\%). This latter effect was marginally significant (one-tailed paired t-test on response proportions after excluding no-response trials, $\mathrm{t}(11)=1.54 ; \mathrm{p}=0.08$ ). In sum, the preTMS results confirm that the participants understood and successfully executed the task.

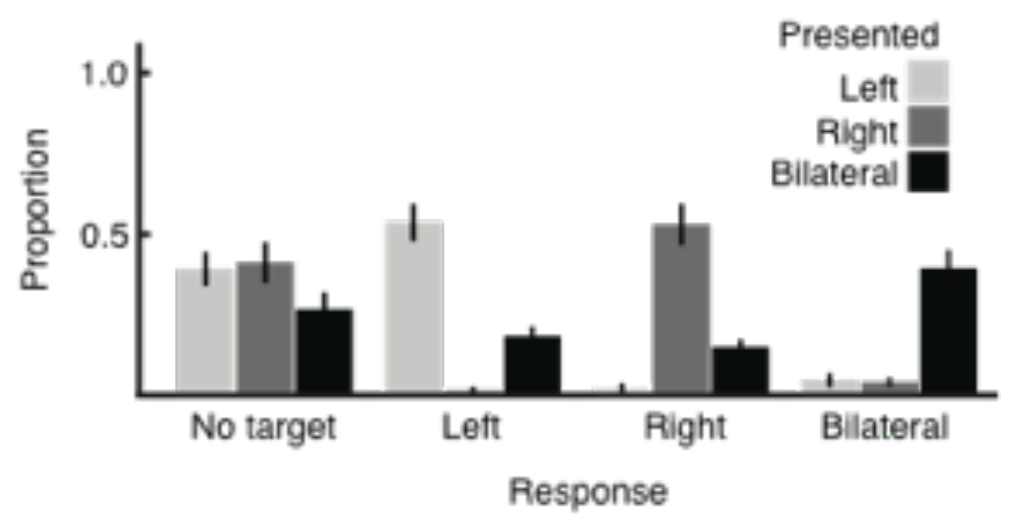

Figure 4: pre-TMS data on target-present trials across future TMS sites. Error bars are \pm 1 SEM.

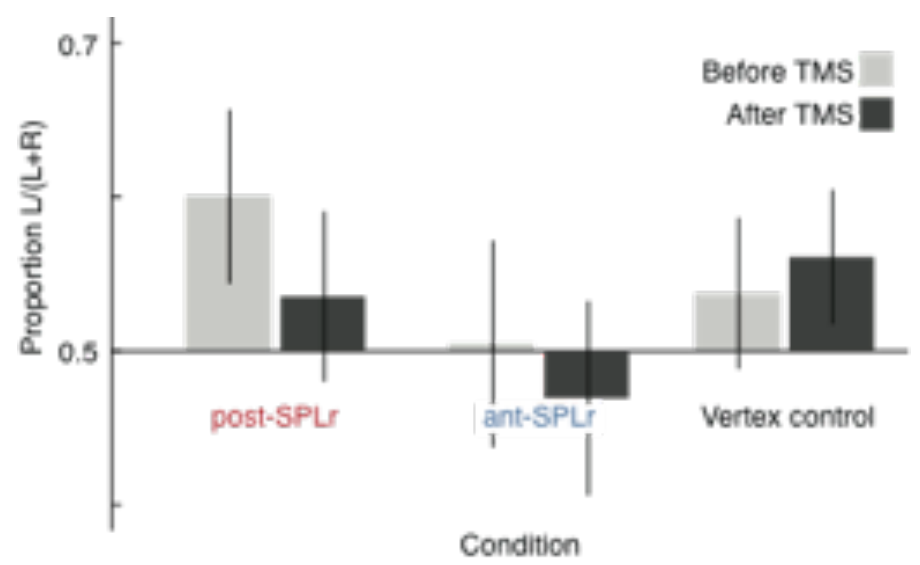

Figure 5: Left/(left and right) response proportion for bilateral targets during task 1, shown for the three test sites, and pre and post TMS respectively. Error bars are \pm 1 SEM. 
We now turn to the effect of TMS. Previous work indicated an shift in bias toward the right visual field following right-parietal TMS (Hilgetag et al., 2001). To investigate whether our data show this same tendency, we focus on the responses to trials with bilateral targets. These are the most informative trials, because the remaining trials are essentially at floor when it comes to erroneous unilateral reports (see Figure 4). For bilateral targets we hypothesise that the slight leftward bias observed before TMS might be reduced afterward. A way of visualising this bias is to calculate the proportion of left responses over the total proportion of unilateral responses, i.e. $\mathrm{L} /(\mathrm{L}+\mathrm{R})$ (see Figure 5). The deviations exceeding 0.5 in the resulting proportions prior to TMS square with the leftward bias reported above. While the effect is not statistically significant, the L/(L/R) ratio numerically decreases following TMS to either parietal site, yet not following TMS to vertex. The direction of this effect would seem to be consistent with our hypothesis, but we are cautious not to over interpret this non-significant result. For each participant we subtracted the $L /(L+R)$ ratios observed pre and post vertex TMS from their $\mathrm{L} / \mathrm{L}+\mathrm{R}$ ratios pre and post TMS to a given parietal site, and entered these normalised ratios into a repeated measures ANOVA using as factors 'pre vs post TMS' and 'parietal stimulation site'. We observed no main effect of 'pre vs pos TMS' $(\mathrm{F}(1,11)$ $=0.75 ; \mathrm{p}=0.41$, indicating that there was no effect compared to vertex even across both parietal sites. The interaction between the two factors was not significant either, $(F(1,11)=0.17 ; p=0.68)$, indicating that the two parietal sites did not differ in their effect on laterality. The analysis above was limited to the trials with bilateral targets, and we investigated whether any effect of parietal TMS would become apparent if we did include unilateral targets as well. We performed a 3-way repeated measures ANOVA analogous to the one above, but now including all target-present trials, leading to factors 'pre vs post TMS', 'parietal stimulation site', as well as 'target location'. Still, neither the main effect of TMS $(F(1,11)=1.02 ; \mathrm{p}=0.33)$ nor the interaction with parietal site reached significance $(F(1,11)=0.29 ; \mathrm{p}=0.60)$.

Conclusions that rest on null results obtained using orthodox statistics are often weak. Conceptually, the absence of a significant result is not evidence towards the absence of an effect. To draw such conclusions, we decided to pursue Bayesian statistics to ascertain how strong the above null results were. As a rule of thumb, Bayes factors between 1 and 3 are considered 'weak' evidence for the null hypothesis, those between 3 and 10 they are considered 'substantial' evidence, and those above 10 are 'strong' (Kass \& Raftery, 1995). First, we revisited the analysis of TMS effects on how participants classified bilateral targets, in particular the 2-way repeated measures ANOVA reported above, with factors 'pre vs post TMS' and 'parietal stimulation site'. We performed Bayesian model comparisons between the model including the factors 'pre vs post TMS', 'parietal stimulation site' and their interaction, and models with factors removed. The Bayes factor of this model comparison was 2.6 in favour of removing the 'pre vs post TMS' factor. This means that, although the model without the effect of TMS explains the data somewhat better, the data do not provide strong evidence that parietal TMS has no effect on this task. We also calculated a Bayes factor of 4.0 in favour of omitting the interaction between 'pre vs post TMS' and 'parietal stimulation site'. This means that the data provide substantial evidence that there is no difference between the parietal sites in their reaction to TMS. Next, we revisited the analysis of TMS effects on how participants classified bilateral as well as unilateral targets. We used the 3-way repeated measures ANOVA reported above, using as factors 'parietal site', 'target location' and 'pre vs post TMS'. The Bayes factor associated with removing the factor 'pre vs post TMS' was 2.7 in favour of the model without any 'pre vs post TMS' effect, again indicating no more than anecdotal evidence for the hypothesis that there is no effect of parietal TMS. The Bayes factor associated with removing the interaction between the factors 'pre vs post TMS' and 'parietal stimulation site' was 4.2, again 
indicating substantial evidence that there is no difference between the parietal sites in their reaction to TMS.

\subsection{Task 2 - Sustained Attention}

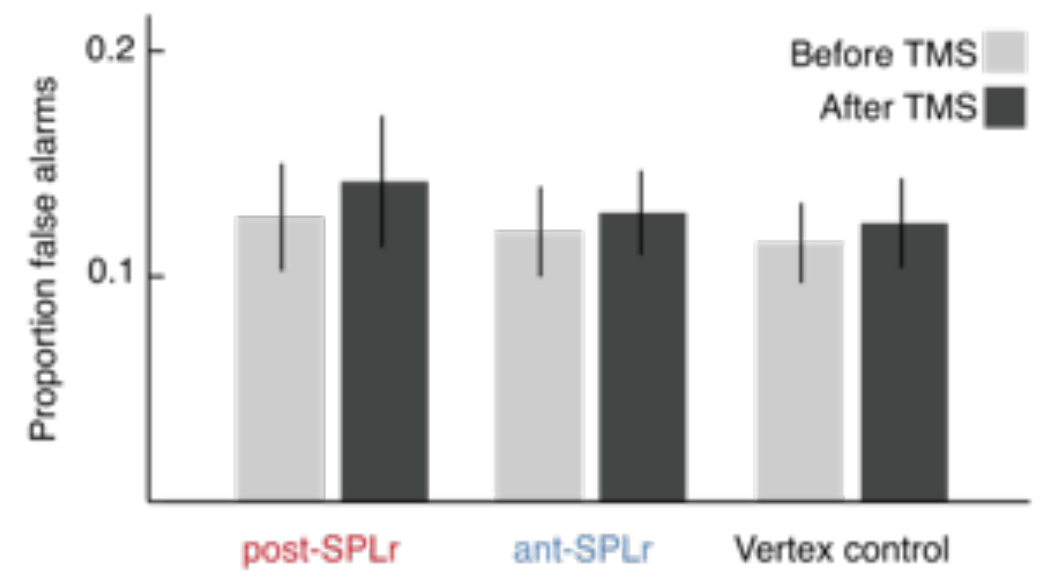

Figure 6: Proportion of false alarms during task 2, shown for the three test sites and pre and post TMS respectively. Error bars are \pm 1 SEM.

For the second task we tested whether the proportion of erroneous responses (i.e. false alarms, or failed response inhibitions) was affected by TMS by comparing this false alarm rate before and after TMS. Figure 6 shows this false alarm rate before and after TMS to each of the three sites. The low overall rate is consistent with the notion that our observers were attentive and motivated to perform the task. It appears that the false alarm rate is increased after TMS, but the figure does not suggest a difference between the three TMS sites this regard. To test this, we entered the false alarm rate into a repeated measures ANOVA using as factors 'TMS site' and 'pre vs post TMS', which indeed revealed no significant main effect of TMS site $(F(1,11)=1.79, p=0.21)$, no main effect of 'pre vs post TMS' $(\mathrm{F}(1,11)=0.41, \mathrm{p}=0.67)$, nor a significant interaction $(\mathrm{F}(1,11)=0.06, \mathrm{p}=0.94)$. This result also appears when we examined the site-specific effect of parietal theta burst TMS by subtracting the site-unspecific vertex effect, analogous to the approach we used in our analysis of task 1. This repeated measured ANOVA on normalised false alarm rates, now with only two levels for 'TMS site', showed no main effect of TMS $(F(1,11)=0.28, p=0.61)$, nor an interaction between TMS and parietal site $(\mathrm{F}(1,11)=0.16, \mathrm{p}=0.69)$. In other words, there is no sign that parietal TMS affects false alarm rate differently than vertex does, nor any sign of a difference between the two parietal sites. These conclusions were supported by Bayes factors. We compared the second ANOVA model above, the one for normalised false alarm rate relative to vertex that used as factors 'TMS site' and 'pre vs post TMS', with a model that did not include the 'pre vs post TMS' factor and found a Bayes factor of 3.5 in favour of that model. In other words, there is substantial evidence for an absence of a differential effect of parietal TMS versus vertex TMS. Similarly, we calculated a Bayes factor of 3.4 in favour of removing the interaction between 'pre vs post TMS' and 'TMS site' in that ANOVA, which strengthens the claim the two parietal sites do not differ in their TMS effect on task performance. 
We had hypothesised that theta burst TMS would lead to a vigilance decrement. Such a decrement can be evidenced, not just as an increased false alarm rate, but also as an increase in the rate at which the incidence of false alarms goes up over time (Malhotra et al.; 2009). We therefore also tested how the incidence of false alarms changed over time in our experiment. To this end we separately assessed the pre-TMS data and post-TMS data associated with each of the three TMS sites, and separated each of these data sets into three equally large time bins, as shown in Figure 7. Visual inspection of data suggests that false alarm rates decrease over the course of time, regardless of whether it was pre or post TMS or which site was stimulated. This most likely reflects training effects, and is consistent with reports of participants, who subjectively felt that their performance improved during the testing sessions. To test this, we performed a 3-way repeated measures ANOVA using the factors 'TMS site', 'time bin' and 'pre vs post TMS'. There was a strong main effect of time bin $(F(2,22)=14.9, p<0.0001)$, but otherwise no significant main effects or interactions $(\mathrm{F}<1.6, \mathrm{~ns})$. This conclusion is again supported by Bayesian statistics. Removal of 'time bin' from the above ANOVA proved detrimental to the model as indicated by a Bayes factor of about 2000 in favour of the full model. The same analysis also showed that removing the interaction of 'pre vs post TMS' and 'TMS site' had a Bayes factor of 12 in favour of the model without the interaction. Finally, removal of the main effect of TMS, namely 'pre vs post TMS', also revealed a Bayes factor of 5.1 in favour of the removal. Together, this is strong evidence towards the claim that TMS has no effect on false alarms in general or over time.
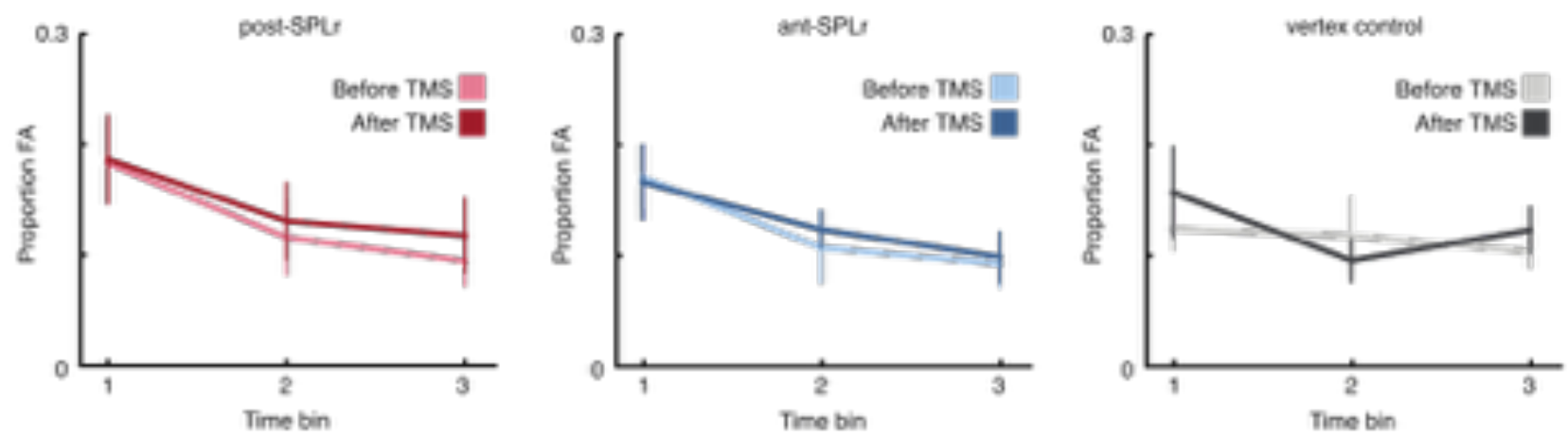

Figure 7: Changes on false alarm rate over time. Red - pre TMS, green - post TMS, blue difference. I) ant-SPLr, II) post-SPLr, III) vertex. Error bars are \pm 1 SEM. 


\section{Discussion}

Opposing effects of TMS over our two right-parietal loci on bistable perception have been replicated several times (Carmel et al., 2010; Kanai et al., 2010, 2011; Wood et al., in prep), yet our current data provide positive evidence, in the form of Bayes factors, that TMS over these loci does not differentially affect the two attention tasks evaluated here. There are several potential explanations for this.

First, it is possible that our TMS methods deviated from the ones employed in previous work on bistable perception. The strongest argument against such an explanation would be to combine, within the same study, a replication of the bistable-perception effects and an evaluation of our attention tasks. In the present study we opted not to attempt such a replication, for three reasons. First, the influence of parietal TMS on bistable perception has now been replicated in five studies that used different inhibitory TMS methodologies. Two such studies used the same offline theta burst protocol employed here (Kanai et al., 2010; 2011) (offline stimulation occurs between experimental recordings, whereas online stimulation takes place during the experimental recording), two others used offline inhibitory repetitive TMS at $1 \mathrm{~Hz}$ (Carmel et al. 2010, Wood et al.), and a fifth one used online repetitive TMS at $2 \mathrm{~Hz}$ (Zaretskaya et al. 2010). Thus, the effects on bistable perception appear robust to methodological variations. Moreover, by directly copying both the neuronavigation and stimulation methods of two of our earlier replications (Kanai et al., 2010; Kanai et al., 2011), we maximised the similarity between the neural effects of the current TMS procedure and those of procedures used in earlier work. Secondly, the sample size we used in this study of 12 was comparable to those used in the previous studies that found positive effects for bistable perception (Carmel et al. 2010: $\mathrm{N}=6$; Kanai et al. 2010: $\mathrm{N}=12$; Zaretskaya, et al., 2010: $\mathrm{N}=15$; Zaretskaya, et al., 2013: $\mathrm{N}=18$; Kanai et al., 2011: $\mathrm{N}=8$; Wood, et al., in prep: $\mathrm{N}($ experiment 1$)=17, \mathrm{~N}($ experiment 2$)=19)$, making us also confident that we would have had the power to replicate the bistable perception result. A third consideration was practical: The inhibitory effects of the cTBS have been reliably observed only in a fairly short temporal window following cTBS application, leaving time for a limited set of conditions. For instance, whereas effects on motor-evoked potentials (MEPs) (Huang et al., 2005) and on EEG synchronisation between the stimulated and contralateral hemisphere (Schindler et al., 2008) were observed up to 60 minutes following stimulation, other studies found influences on saccade latencies for only 30 minutes (Nyffeler et al., 2006), influences on MEP amplitude for 20 minutes (Zafar et al., 2008, using a protocol identical to ours), and influences on the P1-N1 cortical potential for only 16 minutes (Zapallow et al., 2011) following cTBS application. Moreover, mathematical modelling of the data from the study that introduced cTBS (Huang et al., 2005) indicated the inhibitory effect to gradually build up over the first minutes following stimulation (Huang et al., 2011). Conservatively, we therefore decided that in order to securely capture the inhibitory effects of cTBS, the post-TMS experimental recording should start 4 minutes following stimulation and be concluded by 16 minutes following stimulation. In sum, given these time constraints, we decided against the inclusion of a bistable perception condition, as it would have meant spreading conditions across additional cTBS stimulation sessions.

A second potential explanation of the present lack of differential effects of our two TMS manipulations, is that the behavioural tasks were not sensitive enough to pick up changes in attention function that did, in fact, occur. It is certainly true that these two tasks, like all behavioural tasks, do not index all aspects of attention function, so it remains possible that TMS over our two parietal sites had differential effects on an aspect of attention to which our tasks are not sensitive, such as the ability to switch attention between items (Vandenberghe et al., 2001). The data patterns we report, however, do indicate sensitivity with regard to attention functions that are involved in 
performing these two tasks. For instance, we replicate, at a marginally significant level, the leftward tendency that has previously been reported for task 1 (Figures 4 and 5; Hilgetag et al., 2001) and we find robust changes over time in task 2 that are internally consistent across all conditions (Figure 7). Similarly, our pilot work for both tasks was successful in guiding us to difficulty levels where observers' performance was neither at floor nor at ceiling, allowing room for performance changes due to TMS. Finally, our Bayes factor analyses argue against lack of power as an explanation of the observed absence of differential TMS effects.

For the above reasons we view the present data as evidence that the attention functions indexed by our behavioural tasks are not differentially affected by TMS over our two parietal loci and, consequently, that reported differential effects of parietal TMS on bistable perception do not reflect differential effects on sustained and lateralised spatial attention.

Aside from the main question regarding differential effects of TMS to our two parietal loci, we observed minimal evidence for main effects of parietal TMS on our tasks. In the case of lateralised spatial attention (Task 1) we did observe a numerical trend in the direction consistent with existing literature (i.e. for right-parietal TMS to induce an ipsilateral bias; Hilgetag et al., 2001), and Bayes factors did not strongly support the notion that this effect was absent. In the case of sustained attention (Task 2) Bayes factors did indicate the absence of a main TMS effects. Several possibilities may account for this absence of a main effect of parietal TMS in at least one of our two tasks. In the case of task 1, we may simply have stimulated different regions than the TMS study that formed the basis of this task (Hilgetag et al.; 2001). As addressed in the introduction section, assessing the anatomical correspondence between their stimulation site and ours is not straightforward. With regard to task 2 , this task was included, in part, on the basis of lesion studies (e.g. Malhotra et al), and results from such studies need not generalize to TMS work. For instance, lesion-induced neglect or sustained attention impairment may not actually be due to lesions to the parietal cortex in isolation, but emerge as a result of damage to key white matter tracts located beneath parietal cortex. Diffusion tensor imaging suggests that connections between parietal and frontal regions may be damaged in lesion patients displaying deficits in sustained attention (Rueckert \& Grafman, 1996, 1998; Wilkins et al., 1987). It is hence conceivable that any impairment may not be solely due to disruption of the function of individual cortical surface regions in the fronto-parietal network, but rather disconnection between frontal and parietal regions (Bartolomeo et al., 2008, see also Bonelle et al., 2011; Petersen et al., 2012; Husain et al., 1997; Molenberghs et al., 2009; Gaffan \& Hornak, 1997 or Leibovitch et al., 1998 for evidence that lesion-related deficits arise from an interaction with comorbid white matter damage, reviewed in Bartolomeo et al., 2008).

\section{Conclusions}

In sum, while our results are agnostic towards the existence of a parietal parcellation into lateralised spatial and sustained attention regions, we tentatively conclude that the functional fractionation of right-parietal cortex that is observed in bistable perception (Kanai et al., 2011) is unrelated to parietal parcellations that may exist in regard to spatial attention (Yantis et al., 2002; Vandenberghe \& Gillebert, 2009; Müller-Plath et al., 2010) and sustained attention (Pardo et al., 1991; Husain \& Rorden, 2003, Kelley et al., 2007; Malhotra et al., 2009; through review in Vandenberghe et al., 2012). This suggests that the ant-SPLr vs post-SPLr fractionation regarding bistable perception genuinely captures a functional segregation of parietal cortex into regions that specifically perform opposing roles in visual consciousness, rather than attention. 


\section{Acknowledgements}

G. Schauer, R. Kanai and J. Brascamp conceptualised and designed the study. J. Brascamp coded the visual stimuli. G. Schauer and J.Brascamp collected the data. J. Brascamp performed the data analysis. G. Schauer drafted the manuscript, which received critical revisions from J.Brascamp and R. Kanai. All authors approved of the final version of the manuscript for submission. This work was supported by the Japan Science and Technology Agency, the Barbara-Wengeler Foundation as well as the Dr. Mortimer and Theresa Sackler Foundation, which supports the Sackler Centre for Consciousness Science.

\section{References}

Adler, C. M., Sax, K. W., Holland, S. K., Schmithorst, V., Rosenberg, L., \& Strakowski, S. M. (2001). Changes in neuronal activation with increasing attention demand in healthy volunteers: an fMRI study. Synapse, 42(4), 266-272.

Alais, D., van Boxtel, J. J., Parker, A., \& van Ee, R. (2010). Attending to auditory signals slows visual alternations in binocular rivalry. Vision research, 50(10), 929-935.

Bartolomeo, P., De Schotten, M. T., \& Doricchi, F. (2008). Left unilateral neglect as a disconnection syndrome. Cerebral cortex, 17(11), 2479-2490.

Behrmann, M., Ghiselli-Crippa, T., \& Dimatteo, I. (2001). Impaired initiation but not execution of contralesional saccades in hemispatial neglect. Behavioural neurology, 13(1-2), 39-60.

Bjoertomt, O., Cowey, A., \& Walsh, V. (2002). Spatial neglect in near and far space investigated by repetitive transcranial magnetic stimulation. Brain, 125(9), 2012-2022.

Bonnelle, V., Leech, R., Kinnunen, K. M., Ham, T. E., Beckmann, C. F., De Boissezon, X., ... \& Sharp, D. J. (2011). Default mode network connectivity predicts sustained attention deficits after traumatic brain injury. The Journal of Neuroscience, 31(38), 13442-13451.

Brascamp, J., Blake, R., \& Knapen, T. (2015). Negligible fronto-parietal BOLD activity accompanying unreportable switches in bistable perception. Nature neuroscience.

Carmel, D., Walsh, V., Lavie, N., \& Rees, G. (2010). Right parietal TMS shortens dominance durations in binocular rivalry. Current biology, 20(18), R799-R800.

Chambers, C. D., Payne, J. M., Stokes, M. G., \& Mattingley, J. B. (2004). Fast and slow parietal pathways mediate spatial attention. Nature neuroscience, 7(3), 217-218.

Colby, C. L., \& Goldberg, M. E. (1992). The updating of the representation of visual space in parietal cortex by intended eye movements. Science, 255(5040), 90-92.

Corbetta, M., \& Shulman, G. L. (2002). Control of goal-directed and stimulus-driven attention in the brain. Nature reviews neuroscience, 3(3), 201-215.

Corbetta, M., \& Shulman, G. L. (2011). Spatial neglect and attention networks. Annual review of neuroscience, 34, 569.

Critchley, M. (1953). The parietal lobes. London: Edward Arnold. 
Fierro, B., Brighina, F., Oliveri, M., Piazza, A., La Bua, V., Buffa, D., \& Bisiach, E. (2000). Contralateral neglect induced by right posterior parietal rTMS in healthy subjects. Neuroreport, 11(7), 1519-1521.

Gaffan, D., \& Hornak, J. (1997). Visual neglect in the monkey. Representation and disconnection. Brain, 120(9), 1647-1657.

Gainotti, G., D'Erme, P., \& Bartolomeo, P. (1991). Early orientation of attention toward the half space ipsilateral to the lesion in patients with unilateral brain damage. Journal of Neurology, Neurosurgery \& Psychiatry, 54(12), 1082-1089.

Gauthier, L., Dehaut, F., \& Joanette, Y. (1989). The bells test: a quantitative and qualitative test for visual neglect. International Journal of Clinical Neuropsychology.

Heilman, K. M., \& Van Den Abell, T. (1980). Right hemisphere dominance for attention The mechanism underlying hemispheric asymmetries of inattention (neglect). Neurology, 30(3), 327-327.

Heilman, K. M., Watson, R. T., Valenstein, E., \& Damasio, A. R. (1983). Localization of lesions in neglect. Localization in neuropsychology, 33, 471-92.

Hilgetag, C. C., Théoret, H., \& Pascual-Leone, A. (2001). Enhanced visual spatial attention ipsilateral to rTMS-induced'virtual lesions' of human parietal cortex. Nature neuroscience, 4(9), 953-957.

Hodsoll, J., Mevorach, C., \& Humphreys, G. W. (2009). Driven to less distraction: rTMS of the right parietal cortex reduces attentional capture in visual search. Cerebral Cortex, 19(1), 106-114.

Huang, Y. Z., Edwards, M. J., Rounis, E., Bhatia, K. P., \& Rothwell, J. C. (2005). Theta burst stimulation of the human motor cortex. Neuron, 45(2), 201-206.

Huang, Y. Z., Rothwell, J. C., Chen, R. S., Lu, C. S., \& Chuang, W. L. (2011). The theoretical model of theta burst form of repetitive transcranial magnetic stimulation. Clinical Neurophysiology, 122(5), 1011-1018.

Husain, M., \& Nachev, P. (2007). Space and the parietal cortex. Trends in cognitive sciences, 11(1), 30-36.

Husain, M., \& Rorden, C. (2003). Non-spatially lateralized mechanisms in hemispatial neglect. Nature Reviews Neuroscience, 4(1), 26-36.

Husain, M., Shapiro, K., Martin, J., \& Kennard, C. (1997). Abnormal temporal dynamics of visual attention in spatial neglect patients.

Kanai, R., Bahrami, B., \& Rees, G. (2010). Human parietal cortex structure predicts individual differences in perceptual rivalry. Current biology, 20(18), 1626-1630.

Kanai, R., Carmel, D., Bahrami, B., \& Rees, G. (2011). Structural and functional fractionation of right superior parietal cortex in bistable perception. Current biology, 21(3), R106-R107.

Kass, R. E., \& Raftery, A. E. (1995). Bayes factors. Journal of the american statistical association, 90(430), 773-795.

Kelley, T. A., Serences, J. T., Giesbrecht, B., \& Yantis, S. (2007). Cortical mechanisms for shifting and holding visuospatial attention. Cerebral Cortex, 18(1), 114-125. 
Kleinschmidt, A., Büchel, C., Zeki, S., \& Frackowiak, R. S. J. (1998). Human brain activity during spontaneously reversing perception of ambiguous figures. Proceedings of the Royal Society of London. Series B: Biological Sciences, 265(1413), 2427-2433.

Knapen, T., Brascamp, J., Pearson, J., van Ee, R., \& Blake, R. (2011). The role of frontal and parietal brain areas in bistable perception. The Journal of Neuroscience, 31(28), 10293-10301.

Leibovitch, F. S., Black, S. E., Caldwell, C. B., Ebert, P. L., Ehrlich, L. E., \& Szalai, J. P. (1998). Brain-behavior correlations in hemispatial neglect using CT and SPECT The Sunnybrook Stroke Study. Neurology, 50(4), 901-908.

Lumer, E. D., Friston, K. J., \& Rees, G. (1998). Neural correlates of perceptual rivalry in the human brain. Science, 280(5371), 1930-1934.

Malhotra, P., Coulthard, E. J., \& Husain, M. (2009). Role of right posterior parietal cortex in maintaining attention to spatial locations over time. Brain, awn350.

Mesulam, M. (1981). A cortical network for directed attention and unilateral neglect. Annals of neurology, 10(4), 309-325.

Mesulam, M. M. (1985). Attention, confusional states, and neglect. Principles of behavioural neurology, 3, 125-168.

Milner, A. D., \& Goodale, M. A. (1995). The visual brain in action (Vol. 27). Oxford: Oxford University Press.

Molenberghs, P., Gillebert, C. R., Peeters, R., \& Vandenberghe, R. (2008). Convergence between lesion-symptom mapping and functional magnetic resonance imaging of spatially selective attention in the intact brain. The Journal of Neuroscience, 28(13), 3359-3373.

Molenberghs, P., Gillebert, C. R., Schoofs, H., Dupont, P., Peeters, R., \& Vandenberghe, R. (2009). Lesion neuroanatomy of the Sustained Attention to Response task. Neuropsychologia, 47(13), 2866-2875.

Mort, D. J., Malhotra, P., Mannan, S. K., Rorden, C., Pambakian, A., Kennard, C., \& Husain, M. (2003). The anatomy of visual neglect. Brain, 126(9), 1986-1997.

Müller-Plath, G., Ott, D. V., \& Pollmann, S. (2010). Deficits in subprocesses of visual feature search after frontal, parietal, and temporal brain lesions - A modeling approach. Journal of cognitive neuroscience, 22(7), 1399-1424.

Nyffeler, T., Wurtz, P., Lüscher, H. R., Hess, C. W., Senn, W., Pflugshaupt, T., ... \& Müri, R. M. (2006). Repetitive TMS over the human oculomotor cortex: comparison of 1-Hz and theta burst stimulation. Neuroscience letters, 409(1), 57-60.

Paffen, C. L., Alais, D., \& Verstraten, F. A. (2006). Attention speeds binocular rivalry.

Psychological Science, 17(9), 752-756.

Pardo, J. V., Fox, P. T., \& Raichle, M. E. (1991). Localization of a human system for sustained attention by positron emission tomography. Nature, 349(6304), 61-64.

Pastukhov, A., \& Braun, J. (2007). Perceptual reversals need no prompting by attention. Journal of vision, $7(10), 5$. 
Petersen, A., Kyllingsbæk, S., \& Bundesen, C. (2012). Measuring and modeling attentional dwell time. Psychonomic bulletin \& review, 19(6), 1029-1046.

Rees, G., Kreiman, G., \& Koch, C. (2002). Neural correlates of consciousness in humans. Nature Reviews Neuroscience, 3(4), 261-270.

Rice, N. J., Tunik, E., \& Grafton, S. T. (2006). The anterior intraparietal sulcus mediates grasp execution, independent of requirement to update: new insights from transcranial magnetic stimulation. The Journal of neuroscience, 26(31), 8176-8182.

Robertson, I. H., Manly, T., Beschin, N., Daini, R., Haeske-Dewick, H., Hömberg, V., ... \& Weber, E. (1997). Auditory sustained attention is a marker of unilateral spatial neglect. Neuropsychologia, $35(12), 1527-1532$.

Rossi, S., Hallett, M., Rossini, P. M., \& Pascual-Leone, A. (2009). Safety, ethical considerations, and application guidelines for the use of transcranial magnetic stimulation in clinical practice and research. Clinical neurophysiology, 120(12), 2008-2039.

Rueckert, L., \& Grafman, J. (1996). Sustained attention deficits in pat ients with right frontal lesions. Neuropsychologia, 34(10), 953-963.

Rueckert, L., \& Grafman, J. (1998). Sustained attention deficits in patients with lesions of posterior cortex. Neuropsychologia, 36(7), 653-660.

Rushworth, M. F., Ellison, A., \& Walsh, V. (2001). Complementary localization and lateralization of orienting and motor attention. Nature neuroscience, 4(6), 656-661.

Rushworth, M. F. S., \& Taylor, P. C. J. (2006). TMS in the parietal cortex: updating representations for attention and action. Neuropsychologia, 44(13), 2700-2716.

Sack, A. T., Hubl, D., Prvulovic, D., Formisano, E., Jandl, M., Zanella, F. E., ... \& Linden, D. E. J. (2002a). The experimental combination of rTMS and fMRI reveals the functional relevance of parietal cortex for visuospatial functions. Cognitive Brain Research, 13(1), 85-93.

Sack, A. T., Kadosh, R. C., Schuhmann, T., Moerel, M., Walsh, V., \& Goebel, R. (2009). Optimizing functional accuracy of TMS in cognitive studies: a comparison of methods. Journal of Cognitive Neuroscience, 21(2), 207-221.

Sack, A. T., Sperling, J. M., Prvulovic, D., Formisano, E., Goebel, R., Di Salle, F., ... \& Linden, D. E. (2002b). Tracking the mind's image in the brain II: transcranial magnetic stimulation reveals parietal asymmetry in visuospatial imagery. Neuron, 35(1), 195-204.

Samuelsson, H., Hjelmquist, E., Jensen, C., Ekholm, S., \& Blomstrand, C. (1998). Nonlateralized attentional deficits: An important component behind persisting visuospatial neglect?. Journal of Clinical and Experimental Neuropsychology, 20(1), 73-88.

Sarter, M., Givens, B., \& Bruno, J. P. (2001). The cognitive neuroscience of sustained attention: where top-down meets bottom-up. Brain research reviews, 35(2), 146-160.

Schindler, K., Nyffeler, T., Wiest, R., Hauf, M., Mathis, J., Hess, C. W., \& Müri, R. (2008). Theta burst transcranial magnetic stimulation is associated with increased EEG synchronization in the stimulated relative to unstimulated cerebral hemisphere. Neuroscience letters, 436(1), 31-34. 
Serences, J. T., \& Yantis, S. (2007). Spatially selective representations of voluntary and stimulusdriven attentional priority in human occipital, parietal, and frontal cortex. Cerebral Cortex, 17(2), 284-293.

Stone, S. P., Halligan, P. W., \& Greenwood, R. J. (1993). The incidence of neglect phenomena and related disorders in patients with an acute right or left hemisphere stroke. Age and Ageing, 22(1), 46-52.

Thakral, P. P., \& Slotnick, S. D. (2009). The role of parietal cortex during sustained visual spatial attention. Brain Research, 1302, 157-166.

Thut, G., Nietzel, A., \& Pascual-Leone, A. (2005). Dorsal posterior parietal rTMS affects voluntary orienting of visuospatial attention. Cerebral Cortex, 15(5), 628-638.

Vallar, G. (2001). Extrapersonal visual unilateral spatial neglect and its neuroanatomy. Neuroimage, 14(1), S52-S58.

Vandenberghe, R., \& Gillebert, C. R. (2009). Parcellation of parietal cortex: convergence between lesion-symptom mapping and mapping of the intact functioning brain. Behavioural brain research, 199(2), 171-182.

Vandenberghe, R., Gitelman, D. R., Parrish, T. B., \& Mesulam, M. M. (2001). Functional specificity of superior parietal mediation of spatial shifting. Neuroimage, 14(3), 661-673.

Vandenberghe, R., Molenberghs, P., \& Gillebert, C. R. (2012). Spatial attention deficits in humans: the critical role of superior compared to inferior parietal lesions. Neuropsychologia, 50(6), 1092-1103.

Watanabe, T., Masuda, N., Megumi, F., Kanai, R., \& Rees, G. (2014). Energy landscape and dynamics of brain activity during human bistable perception. Nature communications, 5 .

Weilnhammer, V. A., Ludwig, K., Hesselmann, G., \& Sterzer, P. (2013). Frontoparietal cortex mediates perceptual transitions in bistable perception. The Journal of Neuroscience, 33(40), 16009-16015.

Whyte, J., Polansky, M., Fleming, M., Coslett, H. B., \& Cavallucci, C. (1995). Sustained arousal and attention after traumatic brain injury. Neuropsychologia, 33(7), 797-813.

Wilkins, A. J., Shallice, T., \& McCarthy, R. (1987). Frontal lesions and sustained attention. Neuropsychologia, 25(2), 359-365.

Wood, D., Schauer, G., Bak, T., Carmel, D. (in prep). Parietal TMS and binocular rivalry: stimulus and timing dependence resolve contradictory findings.

Yantis, S., Schwarzbach, J., Serences, J. T., Carlson, R. L., Steinmetz, M. A., Pekar, J. J., \& Courtney, S. M. (2002). Transient neural activity in human parietal cortex during spatial attention shifts. Nature neuroscience, 5(10), 995-1002.

Zafar, N., Paulus, W., \& Sommer, M. (2008). Comparative assessment of best conventional with best theta burst repetitive transcranial magnetic stimulation protocols on human motor cortex excitability. Clinical Neurophysiology, 119(6), 1393-1399. 
Zapallow, C. M., Asmussen, M. J., Bolton, D. A., Lee, K. G., Jacobs, M. F., \& Nelson, A. J. (2012). Theta burst repetitive transcranial magnetic stimulation attenuates somatosensory evoked potentials from the lower limb. BMC neuroscience, 13(1), 133.

Zaretskaya, N., Anstis, S., \& Bartels, A. (2013). Parietal cortex mediates conscious perception of illusory gestalt. The Journal of Neuroscience, 33(2), 523-531.

Zaretskaya, N., Thielscher, A., Logothetis, N. K., \& Bartels, A. (2010). Disrupting parietal function prolongs dominance durations in binocular rivalry. Current biology, 20(23), 2106-2111. 